\title{
Regge Slope and the $\Lambda$ Parameter in Quantum Chromodynamics: An Empirical Approach via Quarkonia
}

\author{
W. Buchmüller, G. Grunberg, ${ }^{(a)}$ and S.-H. H. Tye \\ Laboratory of Nuclear Studies, Cornell University, Ithaca, New York 14853
}

(Received 28 April 1980)

\begin{abstract}
The string constant (or, equivalently, the asymptotic Regge slope $\alpha^{\prime}$ ) is related to the $\Lambda$ parameter in quantum chromodynamics (QCD). The empirical information from the $\Psi, \Upsilon$ spectroscopies is used to determine this relation. In so doing a quarkonium potential in excellent agreement with experimental data is also obtained. It is found that $\Lambda_{\text {Trs }}$ $=0.5 \mathrm{GeV}$ and $\alpha^{\prime}=1 \mathrm{GeV}^{-2}$.

PACS numbers: $12.20 . \mathrm{Hx}, 12.40 . \mathrm{Cc}, 12.40 . \mathrm{Mm}$
\end{abstract}

In the past year, a reasonably simple picture of the structures of quantum chromodynamics (QCD) has emerged, mostly due to the works of Creutz, Wilson, ${ }^{1}$ Kogut, Pearson, and Shigemit$\mathrm{su}^{2}$ and Callan, Dashen, and Gross. ${ }^{3}$ It seems that qualitatively different physics appears at different distance scales, which are governed by an effective coupling $g(r)$. At large distances, the string constant $k$ of the strong-coupling limit emerges naturally. At short distances, the only dimensional quantity in QCD (besides quark masses) is the renormalization scale parameter, $\Lambda$, describing the asymptotic-freedom behavior of $g(r)$ in the weak-coupling limit. If quark masses can be neglected, there is only one free parameter in QCD; hence it must be possible to evaluate the relation between $k$ and $\Lambda$.

In fact, the derivation of just such a relation ever, at this moment, none of the methods suggested can give us (in QCD) the relation between $\Lambda$, which has been measured in deep-inelastic scattering experiments, and the Regge slope $\alpha^{\prime}$, as measured in light-hadron spectroscopy. In this Letter, we shall demonstrate that this can be achieved in an empirical way by combining the information from quarkonia (which probe intermediate distances) with the present theoretical understanding of QCD at small and large distances. ${ }^{4}$ In so doing, we obtain a quarkonium potential in excellent agreement with the experimental data.

Let us consider the static potential between a heavy-quark-antiquark pair in the color singlet state. Its three-dimensional Fourier transform is given by (for momentum transfer $\vec{Q}, Q^{2}>0$ )

$$
V\left(Q^{2}\right)=-(4 \pi)^{2} C_{2}(R) \rho\left(Q^{2}\right) / Q^{2} .
$$
has been suggested by various authors. ${ }^{1-3}$ How-

This defines an effective coupling constant $\alpha_{s}\left(Q^{2}\right)$ $\equiv g^{2}\left(Q^{2}\right) / 4 \pi \equiv 4 \pi \rho\left(Q^{2}\right)$. The group factor $C_{2}(R)$ equals $\frac{4}{3}$ in QCD. ${ }^{5}$

At short distances, i.e., $t=Q^{2} / \Lambda^{2}$ large, we have

$$
\rho\left(Q^{2}\right) \sim \frac{1}{b_{0} \ln t}-\frac{b_{1}}{b_{0}} \frac{\ln \ln t}{\left(b_{0} \ln t\right)^{2}}+O\left((\ln t)^{-3}\right),
$$

where $b_{0}=\frac{11}{3} C_{2}(G)-\frac{2}{3} N_{f}$ and

$$
b_{1}=\frac{34}{3}\left[C_{2}(G)\right]^{2}-\frac{10}{3} C_{2}(G) N_{f}-2 C_{2}(R) N_{f}
$$

as given by asymptotic freedom calculations. ${ }^{5}$

The inclusion of $b_{1}$ is necessary to give $\Lambda$ a welldefined interpretation. Here $\Lambda$ is defined in the standard way where the coefficient of the $(\ln t)^{-2}$ term is taken to be zero. At large distances, i.e., $t$ small, linear confinement is expected,

$$
\rho\left(Q^{2}\right) \sim K / Q^{2} .
$$

It is straightforward to check that this implies $V(r) \sim k r$ for large distances. The asymptotic Regge slope is given by $\left(\alpha^{\prime}\right)^{-1}=2 \pi k=4 \pi^{2} C_{2}(R) K$.

The $\beta$ function corresponding to $\rho\left(Q^{2}\right)$ is defined to be $\beta(\rho)=Q^{2}\left(\partial \rho / \partial Q^{2}\right)$. For small values of $\rho$, we have, from Eq. (2),

$$
\frac{b_{0}}{\beta(\rho)}=-\frac{1}{\rho_{2}}\left[1-\frac{b_{1}}{b_{0}} \rho+O\left(\rho^{2}\right)\right] .
$$

For large values of $\rho$, we have, from Eq. (3),

$$
\beta(\rho) \sim-\rho[1+O(1 / \rho)] .
$$

The connection between the Regge slope and the $\Lambda$ parameter requires the knowledge of the $\beta$ function for all values of $\rho, 0 \leqslant \rho<\infty$. In general, we can integrate $\beta(\rho)$ to give $\rho\left(Q^{2}\right)$; in terms of $\Lambda$, we have

$$
\ln \frac{Q^{2}}{\Lambda^{2}}=\frac{1}{b_{0} \rho}+\frac{b_{1}}{b_{0}^{2}} \ln \left(b_{0} \rho\right)+\int_{0}^{\rho} d x\left[\frac{1}{b_{0} x^{2}}-\frac{b_{1}}{b_{0}^{2}} \frac{1}{x}+\frac{1}{\beta(x)}\right],
$$

where the constant of integration [i.e., the $\left(b_{1} / b_{0}^{2}\right) \ln b_{0}$ term] is determined by Eq. (2) for small $\rho$. 
Equation (6) is arranged so that the integral vanishes in the limit as $\rho$ goes to zero. ${ }^{6}$ We can also write $\rho\left(Q^{2}\right)$ as a function of the string constant. Integrating $\beta(\rho)$ in terms of $K$, as given in Eq. (3), we have

$$
\ln \left[\frac{K}{Q^{2}}\right]=\ln \rho+\int_{\rho}^{\infty} d x\left[\frac{1}{x}+\frac{1}{\beta(x)}\right],
$$

where the integral is finite for any positive value of $\rho$ [see Eq. (5)]. To obtain the relation between the Regge slope and the $\Lambda$ parameter measured in deep-inelastic experiments, we observe that

$$
\ln \left(\Lambda / \Lambda_{\overline{\mathrm{ms}}}\right)=\left(1 / 2 b_{0}\right)\left\{\frac{31}{9} C_{2}(G)-\frac{10}{9} N_{f}\right\},
$$

where Eq. (8) is derived by extending the calculations of Susskind and Fischler ${ }^{7}$ to include light fermions. $\Lambda_{\overline{\mathrm{ms}}}$ is related to the $\Lambda_{\mathrm{ms}}$ in the minimal-subtraction scheme ${ }^{8}: \Lambda_{\overline{\mathrm{ms}}}=2.656 \Lambda_{\mathrm{ms}}$. We note that the $Q^{2}$ dependence and the $\rho$ dependence both drop out in the sum of Eqs. (6) and (7). Substituting Eq. (8) into this sum, we obtain the following equation:

$$
\begin{aligned}
-\ln \left(\alpha^{\prime} \Lambda_{\overline{\mathrm{ms}}}^{2}\right)=\ln \left[4 \pi^{2} C_{2}(R)\right]+b_{0}{ }^{-1}\left\{\frac{31}{9} C_{2}(G)\right. & \left.-\frac{10}{9} N_{f}\right\}+\frac{1}{b_{0}}+\frac{b_{1}}{b_{0}^{2}} \ln b_{0} \\
+ & \int_{0}^{1} d x\left[\frac{1}{b_{0} x^{2}}-\frac{b_{1}}{b_{0}^{2} x}+\frac{1}{\beta(x)}\right]+\int_{1}^{\infty} d x\left[\frac{1}{x}+\frac{1}{\beta(x)}\right] .
\end{aligned}
$$

It is clear from Eq. (9) that $\beta(\rho)$ determines the relation between $\alpha^{\prime}$ and $\Lambda_{\overline{\mathrm{ms}}}$. The dimensionless quantity $\alpha^{\prime} \Lambda_{\overline{\mathrm{ms}}}{ }^{2}$ is a function of the gauge group and its fermionic content and does not depend on the value of the coupling in $\mathrm{QCD}$.

In principle, the $\beta$ function can be evaluated directly from QCD. However, this is not possible at the moment. To find a formula for the $\beta$ function that interpolates between the large- $\rho$ [Eq. (5)] and the small- $\rho$ [Eq. (4)] limits, we turn to quarkonium physics which probes the intermediate distance scales. Instead of determining the quarkonium potential and hence $\beta(\rho)$ numerically from the quarkonium data, ${ }^{9}$ we find it more instructive to present a simple empirical formula for the $\beta$ function which (i) satisfies Eqs. (4) and (5), and (ii) provides an excellent fit to the $\Psi$ and $\Upsilon$ data. As an illustration, let us consider

$$
\frac{b_{0}}{\beta(\rho)}=-\frac{1}{\rho^{2}}\left[1-\exp \left(-\frac{1}{b_{0} \rho}\right)\right]^{-1}+\frac{b_{1}}{b_{0}} \frac{1}{\rho} \exp (-l \rho),
$$

where $l$ is a constant. ${ }^{10}$ We note that $\beta$ in Eq. (10) has an essential singularity at $\rho=0$. Such a nonanalytic behavior is compatible with other investigations in QCD. ${ }^{11}$ Equation (10) is shown in Fig. 1.

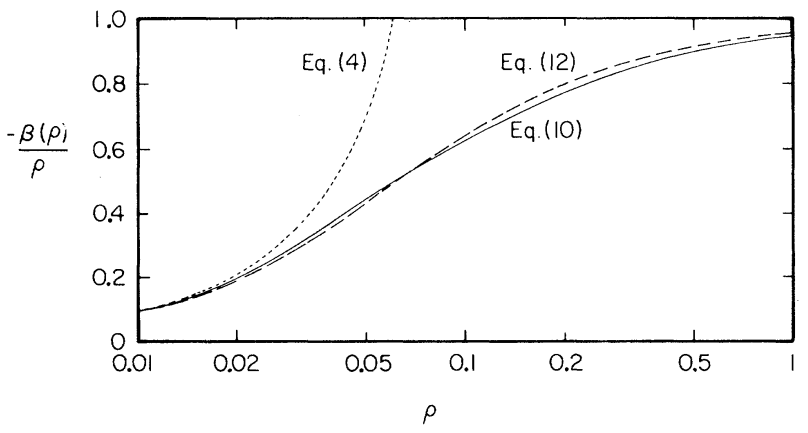

FIG. 1 . The $\beta$ functions: $-\beta(\rho) / \rho$ as a function of $\rho$ for Eq. (4) where higher-order terms are neglected; Eq. (10) with $l=24$; and Eq. (12) with $f=76$.

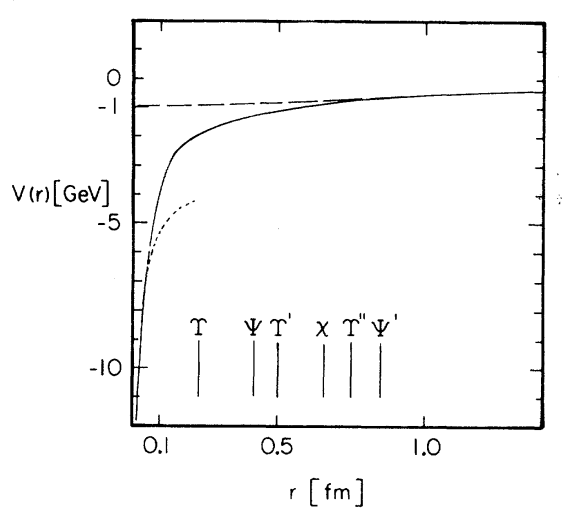

FIG. 2. The potential corresponding to Eq. (10) with $\Lambda_{\overline{\mathrm{ms}}}=0.5 \mathrm{GeV}$. The dotted line represents the "two-loop asymptotic freedom modified" Coulomb potential; the dashed line is the linear potential with $\alpha^{\ell}=1.04 \mathrm{GeV}^{-2}$. The vertical lines indicate the mean square radii of the states. 
Using Eq. (6), we obtain $\rho(t)$ and hence the quarkonium potential. This is plotted in Fig. 2 . Equation (10) also gives $\left(\gamma_{E}=\right.$ Euler's constant $=0.5772$ )

$$
\ln \frac{K}{\Lambda^{2}}=-\ln b_{0}-\frac{b_{1}}{b_{0}^{2}}\left[\gamma_{E}+\ln \left(\frac{l}{b_{0}}\right)\right],
$$

where $b_{0}=9$ and $b_{1}=64$ for QCD with three flavors. [It turns out that the quark momenta in $\Psi$ and $\Upsilon$ satisfy $\left\langle p^{2}\right\rangle<4 m_{c}{ }^{2}$; hence we expect that only three flavors contribute to $V(r)$ ].

From deep-inelastic scattering analysis, ${ }^{12}$ we find $\Lambda_{\overline{\mathrm{ms}}} \leqslant 0.5 \mathrm{GeV}$. Using $\Lambda_{\overline{\mathrm{ms}}}=0.5 \mathrm{GeV}$, we have only one parameter ${ }^{10} l$. We use the $\Upsilon^{\prime}-\Upsilon$ mass difference to fix $l$ while the quark masses are determined by $\Psi$ and $\Upsilon .{ }^{13}$ With $l=24$, we obtain an excellent fit, which is shown in Table I. This also determines the asymptotic Regge slope to be $\alpha^{\prime}=1.0 \mathrm{GeV}^{-2}$. The leptonic widths given in the table are obtained with the Van Royen-Weisskopf formula. ${ }^{14}$ If we naively introduce the $\mathrm{QCD}$ radiative corrections ${ }^{15}$ to the Van Royen-Weisskopf formula, we find that the correction factor [ 1 $\left.-(16 / 3 \pi) \alpha_{s}\right]$ is 0.65 for the $\Upsilon$ states and 0.44 for the $\Psi$ states.

Let us discuss the empirical formula (10) for the $\beta$ function. (i) If we set $b_{1}=0$, the resulting $\beta$ function will give the quarkonium potential that has been suggested by a number of authors. ${ }^{16}$ (ii) If we choose a smaller $\Lambda_{\overline{\mathrm{ms}}}$, say $\Lambda_{\overline{\mathrm{ms}}}=0.4$ $\mathrm{GeV}$, the fit is distinctly worse for any choice of $l$. This is expected since, for small $\Lambda$, the validity of the small-coupling expansion [Eq. (2)] extends to smaller $Q^{2}$ and hence larger distances. However, it is clear that just an "asymptoticfreedom modified" Coulomb potential is not compatible with the $\Psi, \Upsilon$ spectroscopic data. Hence, we expect that for any sensible choice of the $\beta$
TABLE I. The $\Psi$ and $\Upsilon$ spectrum obtained from Eq. (10) with $l=24$. The masses are given in gigaelectronvolts. The leptonic widths under the masses in brackets are given in kiloelectronvolts. They are obtained with use of the Van Royen-Weisskopf formula. $\Psi, \boldsymbol{\Upsilon}$, and $\Upsilon^{\prime}$ masses are inputs (indicated by asterisks).

\begin{tabular}{lcc}
\hline & $\boldsymbol{\Psi}$ & $\boldsymbol{\Upsilon}$ \\
& $m_{c}=1.48 \mathrm{GeV}$ & $m_{\boldsymbol{b}}=4.88 \mathrm{GeV}$ \\
\hline $1 S$ & $3.095^{*}$ & $9.460^{*}$ \\
& $(8.0)$ & $(1.7)$ \\
$1 \boldsymbol{P}$ & 3.52 & 9.89 \\
$2 \boldsymbol{S}$ & 3.69 & $10.02^{*}$ \\
& $(3.6)$ & $(0.76)$ \\
$1 \boldsymbol{D}$ & 3.81 & 10.14 \\
$2 \boldsymbol{P}$ & 3.96 & 10.25 \\
$3 S$ & 4.11 & 10.35 \\
& $(2.5)$ & $(0.54)$ \\
$2 \boldsymbol{D}$ & 4.19 & 10.43 \\
$4 S$ & 4.47 & 10.62 \\
& $(2.0)$ & $(0.45)$ \\
\hline
\end{tabular}

function that satisfies Eq. (4), there is a lower bound on $\Lambda_{\overline{\mathrm{ms}}}$. Our investigation leads us to believe that $\Lambda_{\overline{\mathrm{ms}}}$ must be of the order of a few hundred megaelectronvolts. (iii) On the other hand, we can always find a functional form for the $\beta$ function which fits data and has a very large $\Lambda$. This is because, for very large $\Lambda$, Eq. (2) is valid only for very large $Q^{2}$ or extremely short distances. As a consequence, Eq. (2) or Eq. (4) imposes no constraint on the potential at the distance range which is sensitive to the $\Psi, \Upsilon$ data. Fortunately, deep-inelastic scattering data implies $^{12} \Lambda_{\overline{\mathrm{ms}}} \leqslant 0.5 \mathrm{GeV}$. (iv) Given $\Lambda_{\overline{\mathrm{ms}}} \leqslant 0.5 \mathrm{GeV}$, the $\Psi, \Upsilon$ data are sensitive to the functional form for the $\beta$ function. As an illustration, we consider (see Fig. 1)

$$
\frac{b_{0}}{\beta(\rho)}=-\frac{1}{\rho^{2}}\left[1-\exp \left(-\frac{1}{b_{0} \rho}\right)\right]^{-1}+\frac{b_{1}}{b_{0}} \frac{1}{\rho}\left[1-\exp \left(-\frac{1}{f \rho}\right)\right] .
$$

For any choice of $f$ which satisfies $\Lambda_{\overline{\mathrm{ms}}} \leqslant 0.5$ $\mathrm{GeV}$, this $\beta$ function does not fit data as well as Eq. (10). This implies that the data are very sensitive to the $\beta$ function. We expect that the correct $\beta$ function will be numerically very close to our formula (10), from which we obtain $\alpha^{\prime} \Lambda_{\overline{\mathrm{ms}}}^{2}$ $=0.27, \alpha^{\prime}=1.04 \mathrm{GeV}^{-2}$, and $\Lambda_{\overline{\mathrm{ms}}}=0.508 \mathrm{GeV}$. The uncertainty in $\Lambda_{\overline{\mathrm{ms}}}$ should be no more than 0.1 $\mathrm{GeV}$.

We thank O. Alvarez, K. Gottfried, P. Lepage, S. Rudaz, J. Shigemitsu, T. M. Yan, and D. Yennie for valuable discussions. This research was supported in part by the National Science Foundation, and in part by the German Academic Exchange Service through a NATO Science Fellowship held by one of us (W.B.).

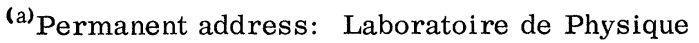
Mathematique, Université des Sciences et Techniques du Languedoc, 34060 Montpellier Cédex, France (Phys-
} 
ique Mathematique et Théorique, equipe de recherche associée au Centre National de la Recherche Scientifique).

${ }^{1}$ M. Creutz, to be published; K. Wilson, Cornell University Report No. CLNS-442, 1980 (to be published). See also.A. Hasenfratz and P. Hasenfratz, CERN Report No. TH 2827, 1980 (to be published).

${ }^{2}$ J. Kogut, R. Pearson, and J. Shigemitsu, Phys. Rev. Lett. 43,484 (1979).

${ }^{3}$ C. G. Callan, R. F. Dashen, and D. J. Gross, Phys. Rev. Lett. 44, 435 (1980), and Phys. Rev. D 20, 3279 (1979).

${ }^{4}$ For earlier attempts in this direction, see, e.g., W. Celmaster and F. S. Henyey, Phys. Rev. D 18, 1688 (1978); R. Levine and Y. Tomozawa, Phys. Rev. D 19, 1572 (1979).

${ }^{5}$ H. D. Politzer, Phys. Rev. Lett. 30, 1346 (1973); D. J. Gross and F. Wilczek, Phys. Rev. Lett. 30, 1343 (1973); W. E. Caswell, Phys. Rev. Lett. 33, 244 (1974); D. R. T. Jones, Nucl. Phys. B75, 531 (1974). Here we follow Gross and Wilczek's notation.

${ }^{6}$ For further discussion of Eq. (6) and its application in perturbative QCD, see G. Grunberg, to be published.

${ }^{7}$ L. Susskind, "Lattice Gauge Theories," Les Houches Lectures, 1976 (to be published). W. Fischler, Nucl. Phys. B129, 157 (1977).

${ }^{8}$ W. A. Bardeen, A. J. Buras, D. W. Duke, and T. Muta, Phys. Rev. D $\underline{18}, 3998$ (1978).
${ }^{9}$ This approach is possible via the inverse-scattering method as suggested by H. B. Thacker, C. Quigg, and J. L. Rosner, Phys. Rev. D 18, 274, 287 (1978), and D 21, 234 (1980).

${ }^{10}$ For small $\rho,-\beta(\rho)=b_{0} \rho^{2}+b_{1} \rho^{3}+b_{2} \rho^{4}+\ldots$ Then $l$ is related to $b_{2}$ by $-b_{1} l=b_{2}-b_{1}{ }^{2} / b_{0}$. However, $b_{2}$ remains to be calculated.

${ }^{11} \mathrm{~J}$. Shigemitsu, private communication.

${ }^{12}$ For a review, see, e.g., A. J. Buras, Rev. Mod. Phys. 52, 199 (1980); J. Ellis, CERN Report No. TH2744-CERN (1979).

${ }^{13}$ For a review of the $\Psi$ data, see, e.g., J. Kirby, in Proceedings of the International Symposium on Lepton and Photon Interactions at High Energies, Batavia, Illinois, 23-29 August 1979 (to be published); D. Andrews et al., Phys. Rev. Lett. 44, 1108 (1980), where earlier references on $\Upsilon$ data can be found.

${ }^{14} \mathrm{R}$. Van Royen and V. Weisskopf, Nuovo Cimento $\underline{50}$, 617 (1967)

${ }^{15}$ R. Barbieri, R. Gatto, R. Kögerler, and Z. Kunszt, Phys. Lett. 57B, 455 (1975); W. Celmaster, Phys. Rev. D 19 , 1517 (1979); E. C. Poggio and H. J. Schnitzer, Phys. Rev. D 20, 1175 (1979).

${ }^{16}$ J. L. Richardson, Phys. Lett. 82B, 272 (1979); R. D. Carlitz and D. B. Creamer, Ann. Phys. (New York) 118, 429 (1979); R. Levine and Y. Tomozawa, Phys. Rev. D 21, 840 (1979). Our choice of Eq. (10) is in part suggested by these works.

\title{
Suppression of the Transverse Scattering Amplitude in ${ }^{207} \mathrm{~Pb}$
}

\author{
C. N. Papanicolas, ${ }^{(a)}$ J. Lichtenstadt, and C. P. Sargent \\ Bates Linear Accelerator Center, Laboratory for Nuclear Science and Department of Physics, \\ Massachusetts Institute of Technology, Cambridge, Massachusetts 02139 \\ and \\ J. Heisenberg \\ University of New Hampshire, Durham, New Hampshire 03824
}

and

J. S. McCarthy

University of Virginia, Charlottesville, Virginia 22901

(Received 11 February 1980)

\begin{abstract}
Transverse form factors have been extracted for the low-lying neutron hole (particle) states of ${ }^{207} \mathrm{~Pb}$ from inelastic-electron-scattering data. A systematic, multipolarityand momentum-transfer-independent quenching of $\sim 55 \%$ in the transverse amplitude is observed when compared with single-particle predictions for both electric and magnetic transitions. The magnitude of the observed effect is not readily explained by our present theoretical understanding of this nucleus.
\end{abstract}

PACS numbers: $25.30 . \mathrm{Cq}, 21.10 . \mathrm{Ky}, 21.10 . \mathrm{Pc}, 27.80 .+\mathrm{w}$

The transverse form factors of the low-lying single-particle (-hole) transitions in ${ }^{207} \mathrm{~Pb}$ have been measured for the first time. Large deviations from the predicted single-particle cross sections have been observed which cannot easily be accommodated within our present understanding of this nucleus in terms of the simple shell model. 